\title{
Investigation of expression and influence of CTGF and HO-1 in rats with diabetic retinopathy
}

\author{
YONGJIAN HUANG $^{1 *}$, CHAOXU QIAN $^{1 *}$, JILIN ZHOU $^{1}$ and JINSONG XUE ${ }^{2}$ \\ ${ }^{1}$ Department of Ophthalmology, The Third People's Hospital of Changzhou, Changzhou, Jiangsu 231001; \\ ${ }^{2}$ Department of Ophthalmology, Nanjing Medical University Affiliated Eye Hospital, Nanjing, Jiangsu 210029, P.R. China
}

Received May 29, 2018; Accepted December 12, 2018

DOI: $10.3892 /$ etm.2019.8395

\begin{abstract}
The expression and influence mechanism of CTGF and HO-1 in rats with diabetic retinopathy (DR) was investigated. One hundred and thirty male Sprague-Dawley (SD) rats were selected and randomly divided into the control group and DR group, with 65 rats in each group. DR was caused by intraperitoneal injection of streptozotocin in rats in the DR group. There were 55 successful models and 10 failed in the modelling. The successful models were sacrificed at the 2nd, 4th and 6th month, respectively. RT-qPCR technology was used for detection of the expression of CTGF and HO-1 in rat retina in each group, $H \& E$ staining for observation of the gradation structure in rat retina and TUNEL method for detection of apoptosis of retinal cells. In the DR group, the retina layers were disordered and a few blood vessels dilated at the 2nd month. In the DR group, the inner membrane of the retina swelled, and the ganglion cells were irregularly arranged at the 4th month. In the DR group, dilatation of the blood vessels was more obvious, the inner membrane edema was more severe, and the arrangement was more irregular at the 6th month. The retinal apoptosis rate of DR rats gradually increased at the $2 \mathrm{nd}$, 4th and 6th month, after which, the CTGF expression gradually increased, but the HO-1 expression gradually decreased in retina in the DR group. However, the mRNA expression of CTGF and HO-1 in the rats at the 2nd, 4th and 6th month in the DR group was higher than that in the control group at the same period. Therefore, CTGF and HO-1 are associated with the occurrence and development of DR in rats and can be considered as targets for the treatment of DR.
\end{abstract}

Correspondence to: Dr Jinsong Xue, Department of Ophthalmology, Nanjing Medical University Affiliated Eye Hospital, 138 Hanzhong Road, Nanjing, Jiangsu 210029, P.R. China E-mail: xhz280@163.com

*Contributed equally

Key words: CTGF, HO-1, diabetic retinopathy, animal model, apoptosis, retina

\section{Introduction}

Diabetic retinopathy (DR) is a microvascular complication caused by diabetes. At present, the prevalence of diabetes in China is approximately $5.6 \%$. Half of the patients with a course of 10-20 years have DR complications, and $100 \%$ of patients with a course of $>20$ years are complicated by DR (1). DR is a chronic progressive irreversible disease with a final outcome of complete loss of vision $(2,3)$. Abnormal insulin in diabetic patients leads to abnormal cell metabolism, damage to the eye nerves, the blood-retinal barrier and microcirculation, and causes fibroplasia and inadequate nutrition supply to the eye, thus contributing to gradual impairment of visual function (4). The pathogenesis of DR is not yet clear, but studies have shown that a variety of factors are associated with the incidence of DR, such as abnormal glucose metabolism, increased blood viscosity, secretion of growth hormone, angiogenesis factor and oxygen free radicals (5). With the advancement of medicine, treatment methods of DR are constantly improving, but so far, almost all the treatments can only reduce the patient's symptoms and slow down the disease process, but cannot reverse it (6). Therefore, investigation of the pathogenesis of DR helps to cure the root cause and look for ways to really treat DR from the source.

CTGF is a connective tissue growth factor and a member of the cysteine growth factor family (7), and its overexpression induces fibrosis, which has been detected in the heart, liver, lung, blood vessels and other tissues $(8,9)$. Under pathological conditions, due to its mitotic characteristics, CTGF is overproduced, which promotes fibroblast proliferation and secretes more ECM, so as to induce fibrosis. Vascular membrane decreases its toughness and is easy to rupture, causing hematocele and retinal detachment. As a result, the visual function is gradually lost and prognosis is poor $(10,11)$.

HO-1 is an oxygen stress-induced heme oxygenase. After the cells are stimulated by stress, MAPK signaling pathway is activated, promotes the translocation of Nrf2 into the nucleus, induces the HO-1 expression, maintains heme concentration in the body and produces biliverdin, $\mathrm{CO}$, and $\mathrm{Fe}^{2+}$, all of which have anti-oxidant and anti-inflammatory effects (12). ERK1/2 is an important signal transduction media for the MAPK signaling pathway, and studies have shown that its phosphorylation can promote CTGF expression (13). Song et al (3) showed that anthocyanin protects retinal cells from oxidative 
stress and inflammation caused by diabetes by regulating $\mathrm{Nrf}$ 2/HO-1 signaling. Therefore, this study aims at providing new ideas for a new and thorough treatment of DR through investigating the expression and influence mechanism of CTGF and $\mathrm{HO}-1$ in DR rats.

\section{Materials and methods}

Experimental animals. One hundred and thirty male SD rats of healthy SPF grade were provided by Shanghai SLAC laboratory Animal Co., Ltd. (Shanghai, China) with a production license of SCXK (Shanghai) 2012-0002. Their age was 8 weeks old and their weight was $150 \pm 30 \mathrm{~g}$. The rats were kept in animal housing of SPF grade, with a temperature of $20 \pm 2^{\circ} \mathrm{C}$, humidity of $60 \pm 5 \%$ and simulated daytime $12 \mathrm{~h}$ and night $12 \mathrm{~h}$. They had free access to basic pellet diet and water intake. The study was approved by the Hospital's Ethics Committee, and the experimental procedures were in accordance with the "Guidelines for the Protection and Use of Laboratory Animals".

Materials. TRIzol kit (Invitrogen; Thermo Fisher Scientific, Inc., Waltham, MA, USA); RT-qPCR random primer (Sangon Biotech Co., Ltd., Shanghai, China); RNase inhibitor (Wuhan Huamei Bioengineering Co., Ltd., Wuhan, China); reverse transcription kit (Life Technologies; Thermo Fisher Scientific, Inc.); fluorescent quantitative PCR kit (Shanghai ShineGene Molecular Biotech, Inc., Shanghai, China); H\&E staining kit (Beyotime Institute of Biotechnology, Haimen, China); TUNEL apoptosis kit (Beijing Jiamei Niunuo Biotechnology Co., Ltd., Beijing, China).

Model building and group processing. After being purchased, the rats were kept for one week, adapted to the new laboratory environment, and randomly divided into the DR and control groups, with 65 rats in each group. Their body weights in the DR group were $135 \pm 31 \mathrm{~g}$, and those in the control group were $140 \pm 33 \mathrm{~g}$. There was no significant difference in the basic data such as weight and age between the two groups ( $\mathrm{P}>0.05)$. After feeding for 4 weeks, each rat was weighed and the streptozotocin (STZ)/saline injection dose was calculated with the rat body weights $(\mathrm{kg})$ x $30 \mathrm{mg}$. Intraperitoneal injection of STZ was performed in the DR group and saline in the control group. To prevent wound infection, every rat received intramuscular injection of penicillin 40,000 units per day for 3 days. Blood was taken from the tail tip of rats for detection of blood glucose concentration at the 5th day. The standard for successful modeling in the DR group was that the blood glucose concentration was $>16.7 \mathrm{mmol} / \mathrm{l}$, and the blood glucose and body weight of the rats were measured monthly. The rats were kept conventionally until the 2nd, 4th and 6th month and sacrificed by excessive anesthesia with intraperitoneal injection of $200 \mathrm{mg} / \mathrm{kg}$ pentobarbital sodium. A total of 55 rats were included in the study. Eighteen rats with successful modeling were sacrificed after the 2nd and 4th month of feeding respectively, and the remaining 19 rats were all sacrificed after the 6th month, and 21, 22 and 22 rats in the control group were sacrificed at the same time.

Detection sample preparation. Complete eyeballs of the rats were taken after sacrifice, and an eyeball of each rat was used to separate the lens and vitreous from the eyecup using an eyeball ring shear. Paraffin sections $(4 \mu \mathrm{m})$ were prepared after $10 \%$ formaldehyde fixed eyecup for HE staining and TUNEL apoptotic cell detection. The eyeball of other rats was used to bluntly separate the retina using ophthalmic microscopy for RT-qPCR detection of the expression of CTGF and HO-1.

Expression of CTGF and $H O-1$ in rat retina after $R T-q P C R$. TRIzol reagent was used to cleave and extract $100 \mathrm{mg}$ of retinal tissues, and the UV spectrophotometer to identify the RNA concentration and purity. OD260/OD280 between 1.8 and 2.0 showed that the specimen purity passed. Preparing $25 \mu \mathrm{l}$ reverse transcription reaction system: $5 \mathrm{X}$ buffer $5 \mu \mathrm{l}$, MMLV (200 U/ $\mu \mathrm{l}) 1 \mu \mathrm{l}$, 4X dNTP (10 mmol/l) $1.25 \mu \mathrm{l}$, RNasin (25 U/ $/ 1$ l) $0.7 \mu \mathrm{l}$, RNase free $\mathrm{H}_{2} \mathrm{O}$ added to $25 \mu \mathrm{l}$. The reverse transcription was performed with PCR instrument at $42^{\circ} \mathrm{C}$ for $15 \mathrm{~min}$ and at $85^{\circ} \mathrm{C}$ for $5 \mathrm{sec}$. The synthesized cDNA amplified according to the prepared $25 \mu \mathrm{l}$ reaction system (SYBR Premix Ex Taq II $12.5 \mu 1$, upstream primer $1 \mu \mathrm{l}$, downstream primer $1 \mu \mathrm{l}$ and 10X cDNA $2 \mu \mathrm{l}$ ) (Table I). The reaction process was a three-step process: the first step was at $95^{\circ} \mathrm{C}$ for $30 \mathrm{sec}$, the second step for a total of 40 cycles, each cycle included $95^{\circ} \mathrm{C}$ for $5 \mathrm{sec}, 60^{\circ} \mathrm{C}$ for $30 \mathrm{sec}$ and $72^{\circ} \mathrm{C}$ for $40 \mathrm{sec}$, and the third step at $72^{\circ} \mathrm{C}$ for $7 \mathrm{~min}$. $\beta$-actin was used as internal reference, and primer sequences were synthesized by Sangon Biotech Co., Ltd. The Ct values obtained by PCR were analyzed and compared, and the relative expression of CTGF and HO-1 was calculated. Experimental results were analyzed using the $2^{-\Delta \Delta \mathrm{Cq}}$ method (14).

$H \& E$ staining. Paraffin sections were deparaffinized and washed 5 times with 95, 75 and 50\% ethanol, respectively. They were washed with distilled water once, then placed in hematoxylin for $5 \mathrm{~min}$ and washed with distilled water again after staining. Paraffin sections were soaked in hydrochloric acid alcohol with a concentration of $1 \%$ for $15 \mathrm{sec}$ to differentiate, and in ammonia water with a concentration of $1 \%$ for $20 \mathrm{sec}$ back to blue after washed with clean water, and in eosin alcohol for 1 min to stain after washed with clean water. Paraffin sections were dehydrated with a low to high concentration gradient of alcohol after being washed with clean water, and soaked in xylene for 2 min for transparency. Histomount was sealed and placed under a light microscope (Olympus, Shanghai, China) for photography and observation.

TUNEL apoptotic cell detection. Paraffin sections were deparaffinized with xylene and alcohol at a gradient concentration, soaked in $50 \mathrm{X}$ proteinase $\mathrm{K}$ for $15 \mathrm{~min}$ at room temperature, and washed twice with PBS, and then TUNEL reaction solution $(50 \mu \mathrm{l})$ was added and incubated in a wet box at $37^{\circ} \mathrm{C}$ incubator for $1 \mathrm{~h}$. After drying the condensed drops of water of the sample, $50 \mu \mathrm{l}$ of POD conversion agent was added and incubation was continued for $30 \mathrm{~min}$. A total of $50 \mu \mathrm{l}$ of DAB solution was added and incubation was continued for $10 \mathrm{~min}$. The steps were the same as the H\&E steps after the incubation, staining, differentiation, back to blue, dehydration, transparency, sealing and light microscopic observation.

The dark brown nucleus was considered to be an apoptotic cell. Each specimen was selected to count the apoptotic 
Table I. Primer sequences.

\begin{tabular}{lll}
\hline Genes & \multicolumn{1}{c}{ Upstream primers } & \multicolumn{1}{c}{ Downstream primers } \\
\hline$\beta$ - actin & 5'-AACCCTAAGGCCAACAGTGAAAAG-3' & 5'-TCATGAGGTAGTCTGTGAGGT-3' \\
$\mathrm{CTGF}$ & 5'-CCGCCAACCGCAAGATT-3' & 5'-CACGGACCCACCGAAGAC-3' \\
$\mathrm{HO}-1$ & 5'-GGAAGAGGAGATAGAGCGAAAC-3' & 5'-AGAGGTCACCCAGGTAGCG-3'
\end{tabular}

cells around the target area and in the center under the light microscope (magnification, $\mathrm{x} 400$ ). The ratio of the number of apoptotic cells to the total number of cells in the visual field was obtained and its average value was the apoptosis rate.

Statistical analysis. The SPSS20.0 [AsiaAnalytics (formerly SPSS China), Shanghai, China] software package was used for processing. The comparison of data between the two groups was performed using KS non-parametric analysis test or t-test based on the distribution characteristics. The comparison of data between groups was performed using ANOVA analysis and Dunnett's post hoc test. The comparison at different time-points in the group was the repeated measures of variance analysis. The significance level is $\alpha=0.05$.

\section{Results}

Body weight and blood glucose monitoring in rats. The experimental results showed that the blood glucose levels in the DR group at the 1st, 2nd, 3rd, 4th, 5th, and 6th month were $22.36 \pm 5.37,23.76 \pm 6.75,26.96 \pm 7.64,25.76 \pm 5.76,27.65 \pm 7.32$ and $25.75 \pm 5.75$, respectively. The blood glucose concentrations in rats in the control group were $5.86 \pm 0.37,4.65 \pm 0.57$, $4.64 \pm 0.47,5.32 \pm 0.63,5.97 \pm 0.75$ and $5.64 \pm 0.62$, respectively. Compared with the control group, the blood sugar level of rats in the DR group significantly increased monthly, while that in the control group was $<6 \mathrm{mmol} / \mathrm{l}$ all the time, within the normal range (all $\mathrm{P}<0.01)$ (Fig. 1). The body weight of rats in the control group increased steadily with the increasing age during the breeding process (all $\mathrm{P}<0.01$ ), while that of the DR group showed a general trend of decline and the monthly body weight was lower than that in the control group $(\mathrm{P}<0.01)$ (Fig. 2).

mRNA expression of CTGF and $\mathrm{HO}-1$ in rat retina. Through detection of the mRNA expression of CTGF and HO-1 after the rats were sacrificed at the $2 \mathrm{nd}, 4$ th and 6 th month, it was indicated that the expression of CTGF mRNA in the DR rats at the 2nd, 4th and 6th month was higher than that in the control group at the same time $(\mathrm{P}<0.01)$; there was no significant fluctuation in the expression of CTGF mRNA at the 2nd, 4th and 6 th month in the control group $(\mathrm{P}>0.05)$. The expression of CTGF mRNA in the DR group gradually increased at the 2nd, 4th and 6th month (all $\mathrm{P}<0.01$ ) (Fig. 3); there was only a small amount of expression of HO-1 mRNA in retina in the control group at the $2 \mathrm{nd}, 4$ th and 6 th month, and there was no statistical difference. The expression of HO-1 mRNA at the 2nd, 4th and 6th month in the DR group was higher than that in the control group, but its expression gradually decreased as time increased (all $\mathrm{P}<0.01$ ) (Fig. 4).

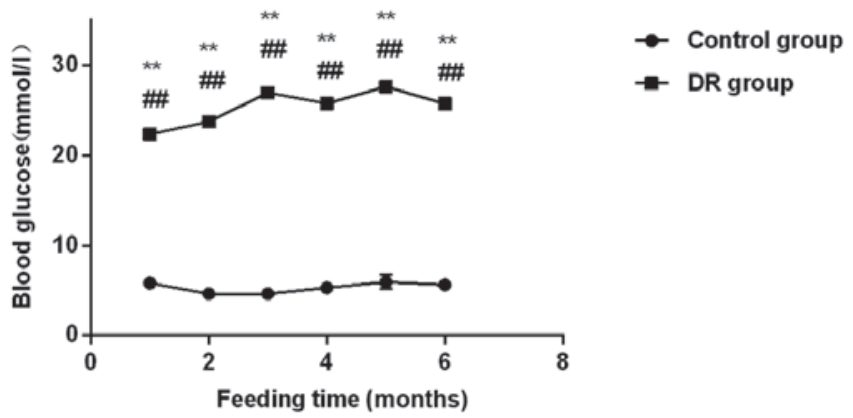

Figure 1. Monthly blood glucose monitoring in rats. The blood glucose level in the DR group significantly increased when compared with the control group $(\mathrm{P}<0.01) .{ }^{* *} \mathrm{P}<0.01$, compared with the control group. ${ }^{\# \#} \mathrm{P}<0.01$, compared with last month. DR, diabetic retinopathy.

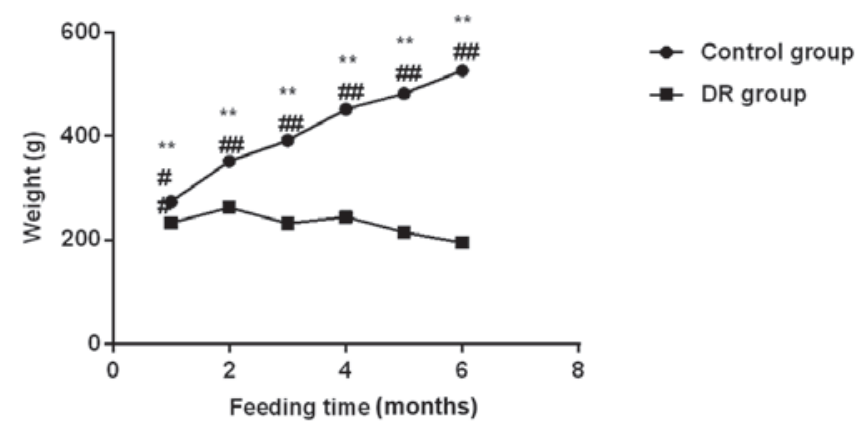

Figure 2. Monthly body weight monitoring in rats. The body weight in the control group gradually increased, and it was higher than that of the previous month from the third month (all $\mathrm{P}<0.01$ ). The body weight in the DR group increased slightly at 2 months, and fluctuated at the 2nd, 4th and 6th month, but the difference was not statistically significant $(\mathrm{P}>0.05)$. The overall body weight in the DR group was still significantly lower than that in the control group $(\mathrm{P}<0.01) .{ }^{* *} \mathrm{P}<0.01$, compared with the control group. ${ }^{\# \#} \mathrm{P}<0.01$, compared with last month. DR, diabetic retinopathy.

Results of $H \& E$ staining. In the control group, the retinal surface of the rats was smooth, with clear layers, complete structure and regular arrangement of cells at the 2nd, 4th and 6th month. In the DR group, the layers of the retina were blurred and a few blood vessels were seen to dilate at the 2 nd month. In the DR group, the inner membrane of the retina showed different sizes of swelling, and the cells were irregularly arranged at the 4th month. In the DR group, most of the blood vessels in the retina dilated and were more obvious, the degree of membrane edema was more severe, and the arrangement was very irregular and almost indistinguishable at the 6th month.

Apoptotic cells detection. Through apoptotic cells detection in rats at the 2 nd, 4 th and 6 th month, it was found that the apoptotic 


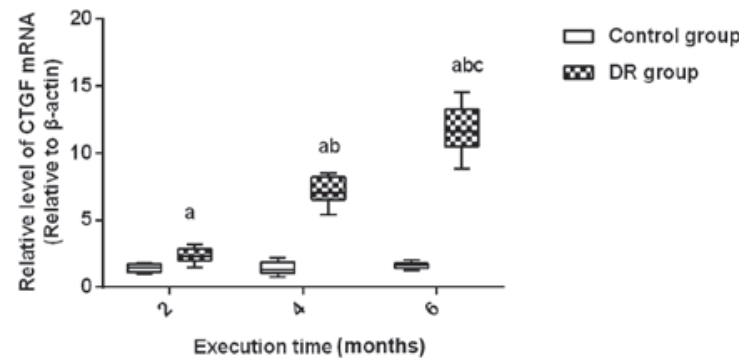

Figure 3. mRNA expression of CTGF in rat retina. RT-qPCR detection indicated that the expression of CTGF mRNA in rats in the DR group at the 2nd, 4th and 6th month was higher than those in the control group at the same time $(\mathrm{P}<0.01)$. There was no significant fluctuation in the expression of CTGF mRNA at the 2nd, 4th and 6th month in the control group ( $\mathrm{P}>0.05)$ The expression of CTGF mRNA in the DR group gradually increased a the 2nd, 4th and 6th month (all $\mathrm{P}<0.01$ ). ${ }^{\mathrm{P}} \mathrm{P}<0.01$, compared with the control group; ${ }^{\mathrm{b}} \mathrm{P}<0.01$, compared with the second month at the same group; ${ }^{\mathrm{C}} \mathrm{P}<0.01$, compared with the 5th month at the same group.

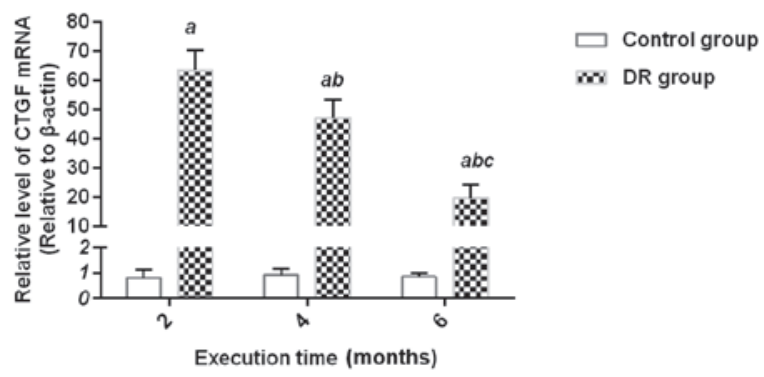

Figure 4. mRNA expression of HO-1 in rat retina. RT-qPCR detection indicated that there was only a small amount of expression of HO-1 mRNA in the retina in the control group at the 2nd, 4th and 6th month, and there was no statistical difference. The expression of HO-1 mRNA at the 2nd, 4th and 6th month in the DR group was higher than that in the control group, but its expression gradually decreased as time increased (all $\mathrm{P}<0.01$ ). ${ }^{\mathrm{P}} \mathrm{P}<0.01$, compared with the control group; ${ }^{\mathrm{b}} \mathrm{P}<0.01$, compared with the $2 \mathrm{nd}$ month at the same group; ${ }^{\mathrm{C}} \mathrm{P}<0.01$, compared with the 5 th month at the same group. DR, diabetic retinopathy.

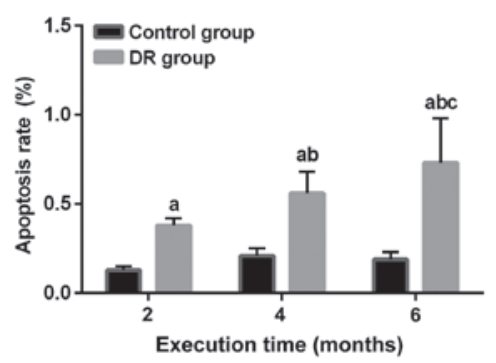

Figure 5. Apoptotic cell detection. TUNEL detection indicated that apoptotic rates of rats in the DR group at the 2nd, 4th and 6th month were higher than those in the control group at the same time $(\mathrm{P}<0.01)$. There was no difference in expression of the apoptotic rate among the 2nd, 4th and 6th month in the control group $(\mathrm{P}>0.05)$. The apoptotic rate in the $\mathrm{DR}$ group increased gradually at the $2 \mathrm{nd}, 4$ th and 6th month (all $\mathrm{P}<0.01$ ). ${ }^{\text {a }} \mathrm{P}<0.01$, compared with the control group; ${ }^{\mathrm{b}} \mathrm{P}<0.01$, compared with the $2 \mathrm{nd}$ month at the same group; ${ }^{\mathrm{c}} \mathrm{P}<0.01$, compared with the 5 th month at the same group. DR, diabetic retinopathy.

rates of rats in the DR group at the 2nd, 4th and 6th month were higher than those in the control group at the same time $(\mathrm{P}<0.01)$. There was no difference in expression of the apoptotic rate among the 2nd, 4 th and 6th month in the control group ( $\mathrm{P}>0.05)$.
In addition, the apoptotic rates in the DR group increased gradually at the 2nd, 4th and 6th month (all $\mathrm{P}<0.01$ ) (Fig. 5).

\section{Discussion}

Diabetes is one of the three major diseases that endanger human physical and mental health in the world today (15). In recent years, with the improvement of people's living standards and the changes of living habits, the incidence of diabetes has been rising year after year, and the number of DR patients has also been increasing each year (16). DR is the result of a combination of factors, and it is chronic, long-term, irreversible, and widely damaging. Its various symptoms and mechanisms are associated with hyperglycemia (17). CTGF is extensively present in the human body, and it can accelerate mitosis, favor cell proliferation, induce apoptosis and have a chemotaxis effect (18). The overexpression of CTGF in certain pathological conditions can lead to a series of pathophysiological processes such as angiogenesis and fibrosis (19). Oxidative stress and high glucose environment can activate the HO-1 expression that can upregulate anti-oxidation and anti-inflammatory factors, thus protecting the retina tissue (20).

In this study, the rats were detected monthly for blood glucose and body weight after successful modeling, the levels of which in the DR group were consistently higher than those in the control group and increased with time. This showed that the DR model we built is successful and can be maintained until the end of the study. Through detection of the mRNA expression of CTGF and HO-1 after the rats were sacrificed at the 2nd, 4th and 6th month, it was shown that those in the DR group at the 2nd, 4th and 6th month were higher than those in the control group at the same time. The expression of CTGF mRNA gradually increased at the 2nd, 4th and 6th month in the DR group. In the analysis of changes in CTGF serum in proliferative DR patients, Baelde et al (21) also found that the CTGF expression increased, which is consistent with this article; while the expression of HO-1 mRNA gradually decreased as time increased. Results of H\&E staining also showed that with the passage of time in the DR group, the retinal swelling degree became more and more severe, the layers were more blurred, the structure incomplete, the arrangement of cells irregular, and the blood vessel dilatation more serious. Apoptotic cell detection showed that the apoptotic rates of rats at the $2 \mathrm{nd}, 4$ th and 6 th month in the DR group were higher than those in the control group at the same time, and they increased gradually at the 2nd, 4th and 6th month in the DR group. Many studies have shown that HO-1 has anti-oxidant and anti-inflammatory protective effects (3), but in this study, detection of the expression in the DR group indicated that it was higher than that in the control group. However, it gradually decreased over time. This may be due to the fact that the HO-1 expression is low under normal conditions, but it increases after oxidative stress and high glucose stress and reaches the peak for a period of time. Over time, this response to stress gradually decreases, so the expression gradually decreases from the peak, and its protective effect gradually weakens. When it is not enough to fight the DR-inducing effect of other genes, the apoptosis of retinal cells gradually increases. At the same time, as diabetes progresses, CTGF gradually increases, more blood vessels are newly formed, fibrosis is induced, and the retina gradually flakes off, exacerbating DR. Sun et al (22) showed that 
the combination of TGF- $\beta 1$ and cerebroside carnosine injection can increase the contents of CTGF and HO-1 in peripheral neuropathy tissue of type 2 diabetes, so as to have a better therapeutic effect. However, in this study, the increase of CTGF promoted the occurrence of DR, because the mitogenicity of CTGF can indeed help to repair tissue, but it promoted fibrosis at the same time, and in the case of retinal tissue, which is particularly required for toughness, this fibrosis undoubtedly damaged the structure and function of the retina.

The conjecture on the role of HO-1 in the occurrence and development of DR and the explanation for the contradiction between the HO-1 expression and the apoptotic rate need to be verified in future experiments, to observe the apoptosis rate of diabetic patients and the retina situation after inhibiting the HO-1 expression. Similarly, the role of CTGF and the above analysis of the results also need similar experiments for verification, and the specific mechanism of signaling pathway needs to be further studied.

In conclusion, CTGF and HO-1 are associated with the occurrence and development of DR in rats. CTGF may induce the occurrence of DR and HO-1 may play a protective role in diabetic retinal tissues. CTGF and HO-1 can be considered as targets for the treatment of DR.

\section{Acknowledgements}

Not applicable.

\section{Funding}

No funding was received.

\section{Availability of data and materials}

The datasets used and/or analyzed during the present study are available from the corresponding author on reasonable request.

\section{Authors' contributions}

YH and CQ performed PCR. JZ and JX were responsible for TUNEL assay. YH and JX helped with H\&E staining. All authors read and approved the final manuscript.

\section{Ethics approval and consent to participate}

The study was approved by the Ethics Committee of The Third People's Hospital of Changzhou (Changzhou, China).

\section{Patient consent for publication}

Not applicable.

\section{Competing interests}

The authors declare that they have no competing interests.

\section{References}

1. Pedersen BK: Anti-inflammatory effects of exercise: Role in diabetes and cardiovascular disease. Eur J Clin Invest 47: 600-611, 2017.
2. Welborn TA, Garcia-Webb P, Bonser A, McCann V and Constable I: Clinical criteria that reflect C-peptide status in idiopathic diabetes. Diabetes Care 6: 315-316, 1983.

3. Song Y, Huang L and Yu J: Effects of blueberry anthocyanins on retinal oxidative stress and inflammation in diabetes through Nrf2/HO-1 signaling. J Neuroimmunol 301: 1-6, 2016.

4. NCD Risk Factor Collaboration (NCD-RisC): Worldwide trends in diabetes since 1980: A pooled analysis of 751 population-based studies with 4.4 million participants. Lancet 387: 1513-1530, 2016.

5. Liu Q, Zhang F, Zhang X, Cheng R, Ma JX, Yi J and Li J: Fenofibrate ameliorates diabetic retinopathy by modulating Nrf2 signaling and NLRP3 inflammasome activation. Mol Cell Biochem: Dec 21, 2017 (Epub ahead of print).

6. Keech AC, Mitchell P, Summanen PA, O'Day J, Davis TM, Moffitt MS, Taskinen MR, Simes RJ, Tse D, Williamson E, et al; FIELD study investigators: Effect of fenofibrate on the need for laser treatment for diabetic retinopathy (FIELD study): A randomised controlled trial. Lancet 370: 1687-1697, 2007.

7. Henshaw FR, Boughton P, Lo L, McLennan SV and Twigg SM: Topically applied connective tissue growth factor/CCN2 improves diabetic preclinical cutaneous wound healing: Potential role for CTGF in human diabetic foot ulcer healing. J Diabetes Res 2015: 236238, 2015.

8. Liu F, Tang W, Chen D, Li M, Gao Y, Zheng H and Chen S: Expression of TGF- $\beta 1$ and CTGF is associated with fibrosis of denervated sternocleidomastoid muscles in mice. Tohoku J Exp Med 238: 49-56, 2016

9. Montford JR and Furgeson SB: A new CTGF target in renal fibrosis. Kidney Int 92: 784-786, 2017.

10. Klaassen I, van Geest RJ, Kuiper EJ, van Noorden CJ and Schlingemann RO: The role of CTGF in diabetic retinopathy. Exp Eye Res 133: 37-48, 2015.

11. Zhang H, Cai X, Yi B, Huang J, Wang J and Sun J: Correlation of CTGF gene promoter methylation with CTGF expression in type 2 diabetes mellitus with or without nephropathy. Mol Med Rep 9: 2138-2144, 2014

12. Liu J, Wang L, Tian XY, Liu L, Wong WT, Zhang Y, Han QB, Ho HM, Wang N, Wong SL, et al: Unconjugated bilirubin mediates heme oxygenase-1-induced vascular benefits in diabetic mice. Diabetes 64: 1564-1575, 2015.

13. Chen YC, Chen BC, Yu CC, Lin SH and Lin CH: miR-19a, -19b, and -26b mediate CTGF expression and pulmonary fibroblast differentiation. J Cell Physiol 231: 2236-2248, 2016.

14. Livak KJ and Schmittgen TD: Analysis of relative gene expression data using real-time quantitative PCR and the 2(-Delta Delta C(T)) method. Methods 25: 402-408, 2001.

15. Zinman B, Lachin JM and Inzucchi SE: Empagliflozin, cardiovascular outcomes, and mortality in type 2 diabetes. $\mathrm{N}$ Engl $\mathrm{J}$ Med 374: 1094, 2016.

16. Pothineni NV and Mehta JL: Follow-up of glycemic control and cardiovascular outcomes in type 2 diabetes. N Engl J Med 373: 977-978, 2015.

17. Piette JD, Heisler $M$ and Wagner TH: Problems paying out-of-pocket medication costs among older adults with diabetes. Diabetes Care 27: 384-391, 2004.

18. Wang J, Duan L, Guo T, Gao Y, Tian L, Liu J, Wang S and Yang J: Downregulation of miR-30c promotes renal fibrosis by target CTGF in diabetic nephropathy. J Diabetes Complications 30: 406-414, 2016.

19. Zhu L, Zhao S, Liu S, Liu Q, Li F and Hao J: PTEN regulates renal extracellular matrix deposit via increased CTGF in diabetes mellitus. J Cell Biochem 117: 1187-1198, 2016.

20. Negi G, Nakkina V, Kamble P and Sharma SS: Heme oxygenase-1, a novel target for the treatment of diabetic complications: Focus on diabetic peripheral neuropathy. Pharmacol Res 102: 158-167, 2015.

21. Baelde HJ, Eikmans M, Lappin DW, Doran PP, Hohenadel D, Brinkkoetter PT, van der Woude FJ, Waldherr R, Rabelink TJ, de Heer E, et al: Reduction of VEGF-A and CTGF expression in diabetic nephropathy is associated with podocyte loss. Kidney Int 71: 637-645, 2007

22. Sun J, Zheng H, Qin X and Qi L: Effects of immunocytokine combined with cattle encephalon glycoside and ignotin on CTGF, HO-1 and NT-3 in patients with type 2 diabetic peripheral neuropathy. Iran J Public Health 46: 1632-1638, 2017. 